\title{
Thickness-dependent stress effect in p-type metal-oxide-semiconductor structure investigated by substrate injection current
}

\author{
Chao-Chi Hong, Wei-Jian Liao, and Jenn-Gwo Hwu ${ }^{\text {a) }}$ \\ Department of Electrical Engineering/Graduate Institute of Electronics Engineering, National Taiwan \\ University, Room 446, Taipei, Taiwan, Republic of China
}

(Received 28 October 2002; accepted 4 April 2003)

\begin{abstract}
The effects of oxide, $\mathrm{Si}$ wafer, and gate $\mathrm{Al}$ thicknesses on the substrate injection currents $\left(J_{\text {sub }}\right)$ of $p$-type metal-oxide-semiconductor structures with ultrathin oxides are studied. $J_{\text {sub }}$ is reported to be both trap-related (interface and Si bulk) and Si band gap-related (intrinsic carrier concentration). Both mechanisms have given rise to the stress near the $\mathrm{Si} / \mathrm{SiO}_{2}$ interface. Current-voltage and capacitance-voltage characterizations reveal that $J_{\text {sub }}$ increases with oxide thickness, which is suggested to be dominated by the trap-related mechanism. A stronger dependence of $J_{\text {sub }}$ on a change in oxide thickness is observed for a thicker Si wafer, which is proposed to be mainly caused by the band gap-related mechanism. Furthermore, a thicker gate $\mathrm{Al}$ introduces a higher $J_{\text {sub }}$, which is proposed to be due to both the trap-related and the band gap-related mechanisms. (c) 2003 American Institute of Physics. [DOI: 10.1063/1.1581004]
\end{abstract}

Stress-related problems have become pervasive in the complementary metal-oxide-semiconductor technology. Many device failures are caused by the stress that occurs during various processing stages. ${ }^{1}$ Thermal oxidation of silicon requires volume expansion, and the oxidation rate depends strongly on the geometry ${ }^{2}$ and external stress. ${ }^{3}$ Multiple overlaid films with different elastic and thermal properties introduce film stress, ${ }^{4}$ which is especially large at the edges of the films. ${ }^{5}$

Many electrical parameters of the metal-oxidesemiconductor (MOS) field-effect transistor (MOSFET) have been reported to be affected by the process-induced stress. For example, mechanical stress which is induced by the gate dielectrics ${ }^{6}$ influences the interface trap density, causing a threshold voltage shift, ${ }^{7}$ increasing the cumulative failure rate of time-dependent dielectric breakdown. ${ }^{8}$

$\mathrm{Ma}$ and co-workers studied the dependencies of radiation-induced ${ }^{9}$ and hot carrier-induced effects ${ }^{10}$ on the thickness of gate $\mathrm{Al}$ in MOS capacitors. However, the relationship between the substrate injection current $\left(J_{\text {sub }}\right)$ and the thickness of gate $\mathrm{Al}$ of MOS capacitors is still unknown. $J_{\text {sub }}$ of MOS capacitors has been stated to be useful in the Si-based photodetector. ${ }^{11}$ Besides, the dependency of $J_{\text {sub }}$ on the thicknesses of the oxide and the silicon wafer in an MOS structure is of interest. Thus, this work considers not only gate $\mathrm{Al}$, but also the oxide and $\mathrm{Si}$ wafer thicknesses, with reference to the change in $J_{\text {sub }}$ of MOS capacitors.

$p$-type 3 in. (100) Si wafers with a resistivity of 1-10 $\Omega \mathrm{cm}$ were used. After standard Radio Corporation of America cleaning, several thick $(500 \mu \mathrm{m})$ and thin $(100 \mu \mathrm{m})$ silicon wafers were oxidized in a rapid thermal processor at $850{ }^{\circ} \mathrm{C}, 50$ Torr of $\mathrm{O}_{2}$, for various times to obtain oxides with different thicknesses. When comparing the effects of wafer thickness, the thickness of gate $\mathrm{Al}$ was fixed at 3000 $\AA$. In the experiments on the effects of gate $\mathrm{Al}$ thickness, 500

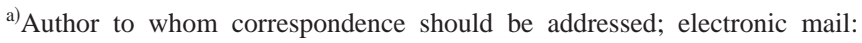
hwu@cc.ee.ntu.edu.tw
}

$\mu \mathrm{m}$ thick wafers were used and the thickness of gate $\mathrm{Al}$ was set to $4000 \AA, 6000 \AA$, and $8000 \AA$, on different chips. After gate $\mathrm{Al}$ deposition, MOS capacitors of size $150 \mu \mathrm{m}$ by 150 $\mu \mathrm{m}$ were formed by conventional photolithography. Current-voltage $(I-V)$ and capacitance-voltage $(C-V)$ curves were measured with an HP 4154 and an HP 4286, respectively. A $100 \mathrm{~W}$ halogen tube was employed as the light source to take $C-V$ measurements under illumination.

Figure 1 shows the gate $\left(J_{g}\right)$ and substrate injection current densities $\left(J_{\text {sub }}\right)$ of MOS capacitors with various optical oxide thicknesses, with the wafer and gate $\mathrm{Al}$ thicknesses fixed at $500 \mu \mathrm{m}$ and $3000 \AA$, respectively. Intuitively, $J_{g}$ decreases with the oxide thickness. On the other hand, $J_{\text {sub }}$ increased with the oxide thickness. The inset presents a typical $I-V$ curve, showing that $J_{\text {sub }}$ saturates when gate voltage $>2 \mathrm{~V}$ under inversion region. $J_{\text {sub }}$ is limited by the slow supply of minority carriers, electrons, in the depletion region, and the detailed mechanism will be discussed in the next paragraph. The current saturation phenomenon under inversion cannot be easily observed in MOSFETs and $n$-type MOS capacitors. In MOSFETs, the minority carrier can be

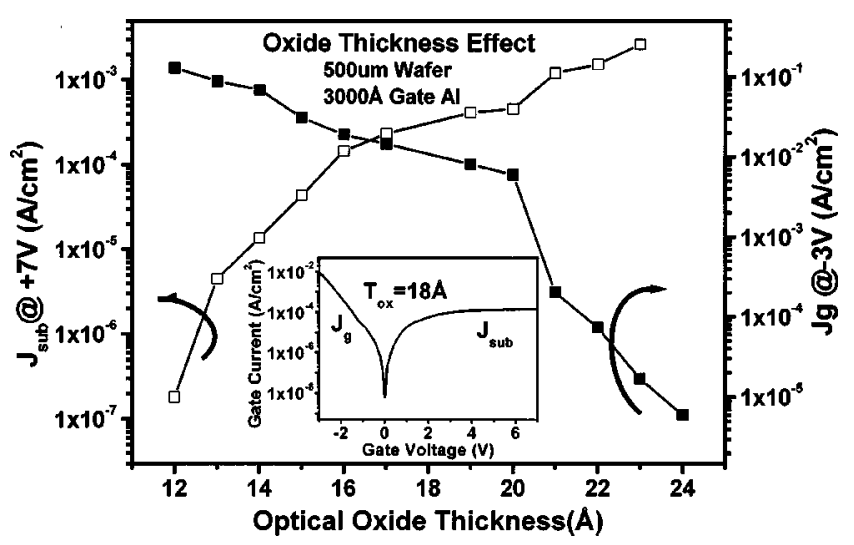

FIG. 1. $J_{\text {sub }}$ and $J_{g}$ of $p$-type MOS structures with oxides of various optical thicknesses. The inset shows a representative $I-V$ curve of a device with 18 A optical oxide thickness. 


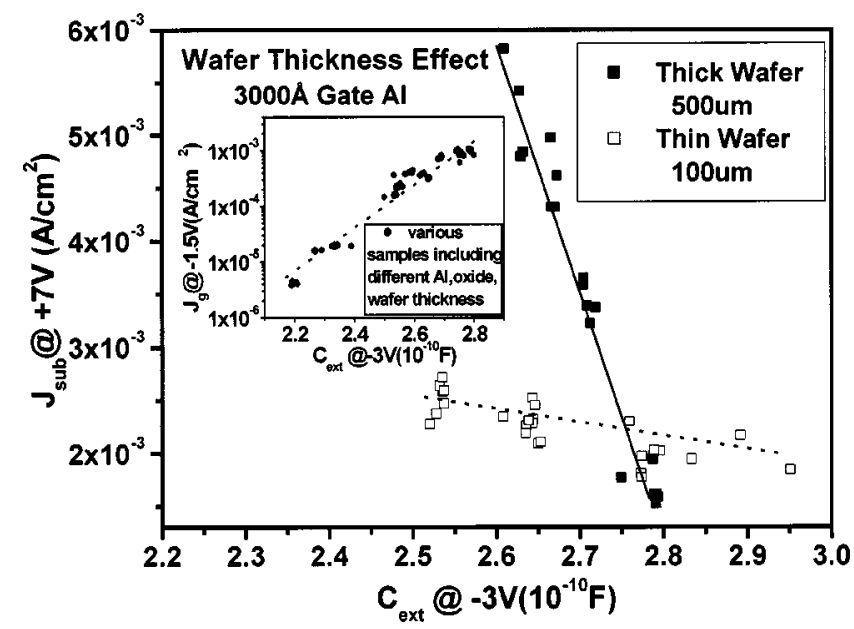

FIG. 2. The $J_{\text {sub }}$ vs $C_{\text {ext }}$ of samples on the thick and thin silicon wafers. The inset shows $J_{g}$ versus extracted capacitances for various samples in this work including different wafer, oxide, and gate Al thicknesses.

supplied via the source and drain regions. ${ }^{12}$ In $n$-type MOS capacitors, the direct tunneling current of the majority electron carriers from the metal to the silicon is high under the inversion region, because the barrier height for electrons to tunnel though the oxide layer is lower. ${ }^{13}$ Accordingly, the limitation of the supply of minority hole carriers is unobservable in such a case.

Reportedly, $J_{\text {sub }}$ of $p$-type MOS capacitors involve two major components; the depletion region generation current $\left(J_{\mathrm{dg}}\right)$, and the interface states generation current $\left(J_{\mathrm{sg}}\right) .{ }^{14-16}$ $J_{\text {dg }}$ increases with $\mathrm{Si}$ bulk trap density, which relates to the minority carrier lifetime $\left(\tau_{n}\right) . J_{\mathrm{sg}}$ increases with the interface states density $\left(D_{\text {it }}\right)$. The mechanism for which $J_{\text {sub }}$ changes with $\tau_{n}$ and $D_{\text {it }}$, is called the trap-related effect in this letter. In addition, both $J_{\mathrm{dg}}$ and $J_{\mathrm{sg}}$ increase with $\mathrm{Si}$ intrinsic carrier concentration $\left(n_{i}\right)$, which is governed by the band gaprelated effect caused by the external stress. The Si band gap decreases (increases) when the Si lattice is under compressive (tensile) stress, ${ }^{17}$ increasing (decreasing) $n_{i}$ and thus $J_{\text {sub }}$.

The mechanism for which $J_{\text {sub }}$ increases with the oxide thickness in Fig. 1 is investigated as follows. The Si wafer undergoes tensile stress after oxidation due to volume expansion, and a thicker oxide may apply a larger tensile stress to the Si wafer, increasing the band gap, and decreasing $n_{i}$ and thus $J_{\text {sub }}$. However, experimental data indicate that $J_{\text {sub }}$ increases with the oxide thickness. Probably, the Si bondings near the $\mathrm{Si} / \mathrm{SiO}_{2}$ interface are broken after oxidation to create the Si interface and bulk traps, releasing some of the tensile stress, such that the band gap-related effect becomes small. However, a thicker oxide consumes a larger volume of $\mathrm{Si}$, which may contribute to higher interface and bulk trap densities, increasing $J_{\text {sub }}{ }^{18}$ So the trap-related effect dominates the $J_{\text {sub }}$ behavior of devices for different oxide thicknesses.

Figure 2 shows the dependencies of $J_{\text {sub }}$ and the extracted gate capacitance $\left(C_{\text {ext }}\right)$ of the devices on the thick and thin wafers. $C_{\text {ext }}$ is obtained from the two-frequency correction method developed by $\mathrm{Hu} .{ }^{19}$ The purpose of converting the optical oxide thickness to $C_{\text {ext }}$ is to more precisely determine the thickness of thin oxides. A larger $C_{\text {ext }}$ under accumulation regions means a thinner oxide. The inset

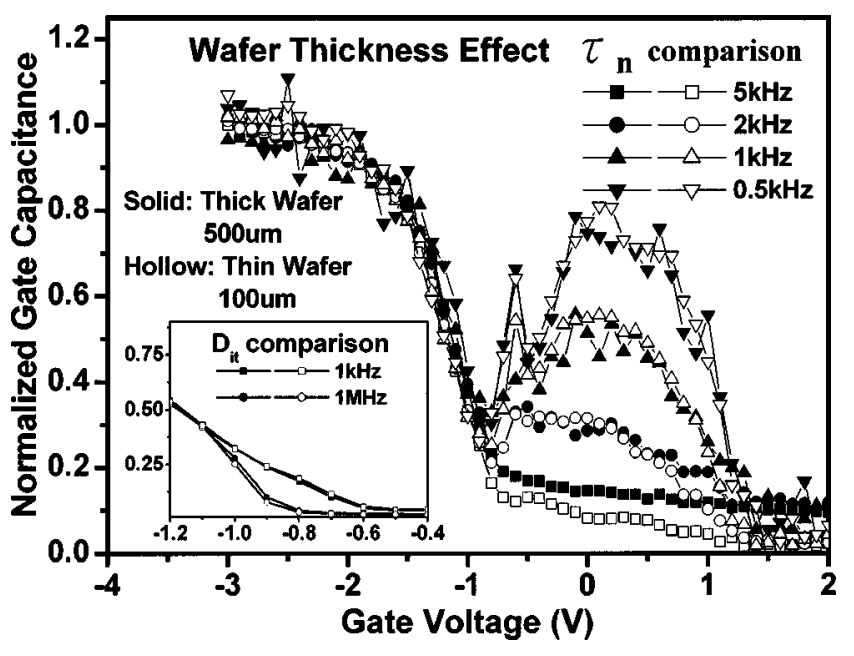

FIG. 3. The normalized $C-V$ curves measured under illumination at various frequencies for samples on the thick and thin wafers, using for $\tau_{n}$ comparison. The inset shows dark $C-V$ curves in depletion region measured at two different frequencies, using for $D_{\text {it }}$ comparison.

in Fig. 2 supports the claim that $J_{g}$ increases with $C_{\text {ext }}$ for the various samples used in this work, including those with different gate $\mathrm{Al}$, oxide, and silicon wafer thicknesses. $J_{\text {sub }}$ of the devices on both the thick and thin wafer falls as $C_{\text {ext }}$ increases; that is, it increases with oxide thickness, consistent with Fig. 1. However, the slopes of the solid and hollow data point distributions clearly differ from each other. $J_{\text {sub }}$ of devices on the thick wafer are more sensitive to the change in oxide thickness than that of those on the thin wafer.

$C-V$ measurements were made to determine the $J_{\text {sub }}$ composition. The magnitude of inversion capacitance at a given frequency provides information on $\tau_{n}$, and therefore, on the bulk trap density; a comparison of capacitance values at two different frequencies under the depletion region provides the information on the $D_{\text {it }} \cdot{ }^{13,20}$

Here, $C-V$ measurements were made on one device on the thick wafer and the other on the thin wafer in Fig. 2, whose $C_{\text {ext }}$ at $-3 \mathrm{~V}$ are both $2.6 \times 10^{-10} \mathrm{~F}$. Figure 3 shows the results. Illumination was made during the measurements of $\tau_{n}$ comparisons to increase the supply of minority carrier. Figure 2 clearly shows that devices on the thick wafer exhibit higher $J_{\text {sub }}$ than those on the thin wafer at a $-3 \mathrm{~V} C_{\text {ext }}$ value of about $2.6 \times 10^{-10} \mathrm{~F}$. However, the $C-V$ curves in Fig. 3 and its inset indicate that both $\tau_{n}$ and $D_{\text {it }}$ differ little between the devices on the thick and thin wafers. Hence, the different sensitivities of $J_{\text {sub }}$ to the change in $C_{\text {ext }}$ (oxide thickness) between devices on the thick and thin wafers are not caused by the trap-related effect.

The ideal ratio of the stress sustained by silicon to that by oxide in an MOS structure is, ${ }^{21}$

$$
\sigma_{\mathrm{Si}} / d_{\text {oxide }}=4 \sigma_{\text {oxide }} / d_{\mathrm{Si}}
$$

where $\sigma_{\mathrm{Si}}$ and $\sigma_{\text {oxide }}$ are the average stresses of $\mathrm{Si}$ and oxide, and $d_{\mathrm{Si}}$ and $d_{\text {oxide }}$ are the thicknesses of the Si wafer and the oxide, respectively. Clearly, different $\mathrm{Si}$ wafer thicknesses induce different $\mathrm{Si}$ stress changes for a given particular change in the oxide thickness. As a result, the Si band gap, $n_{i}$, and then $J_{\text {sub }}$ of devices on the thick and thin wafers have different sensitivities to the change in the oxide thickness. 


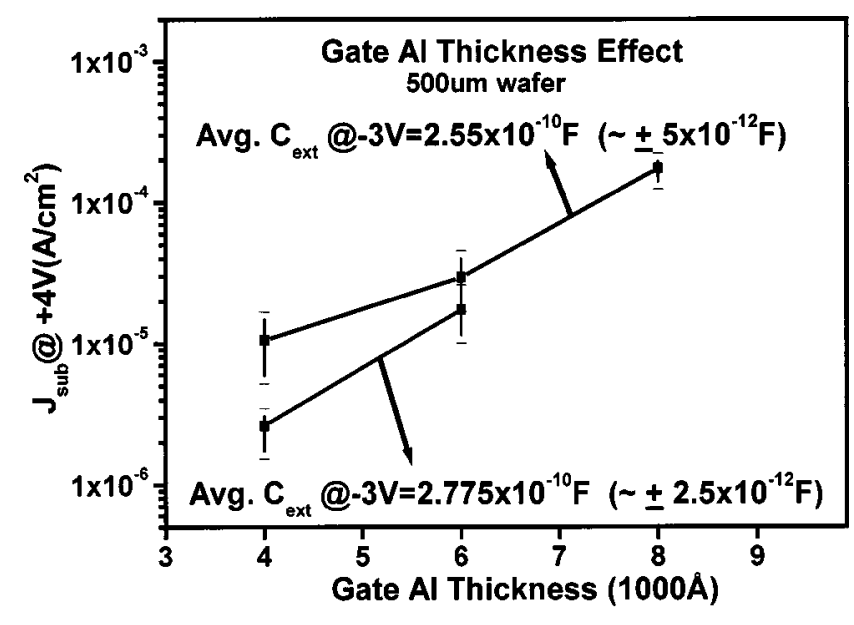

FIG. 4. The $J_{\text {sub }}$ of $p$-type MOS structures versus gate Al thicknesses for samples under two different $C_{\text {ext }}$.

The mechanism for which Si wafer thickness affect $J_{\text {sub }}$ is supposed to be caused mainly by the band gap-related effect.

Figure 4 plots the effects of gate $\mathrm{Al}$ thickness on $J_{\text {sub }}$ of devices with two similar $C_{\text {ext }}$ values on a $500 \mu \mathrm{m} \mathrm{Si} \mathrm{wafer.}$ For a given wafer thickness and similar $C_{\text {ext }}$ (oxide thickness), a thicker gate Al thickness yields a higher $J_{\text {sub }}$. Again $C-V$ analysis was employed and the results are shown in Fig. 5. First, the inset of Fig. 5 shows $C-V$ curves under the depletion region, indicating little difference among the $D_{\text {it }}$ values for all samples. Second, the magnitude of inversion capacitance at a certain frequency increases with gate $\mathrm{Al}$ thickness. Consequently, a thicker gate Al corresponds to a shorter $\tau_{n}$ and therefore, a higher the Si bulk trap density,

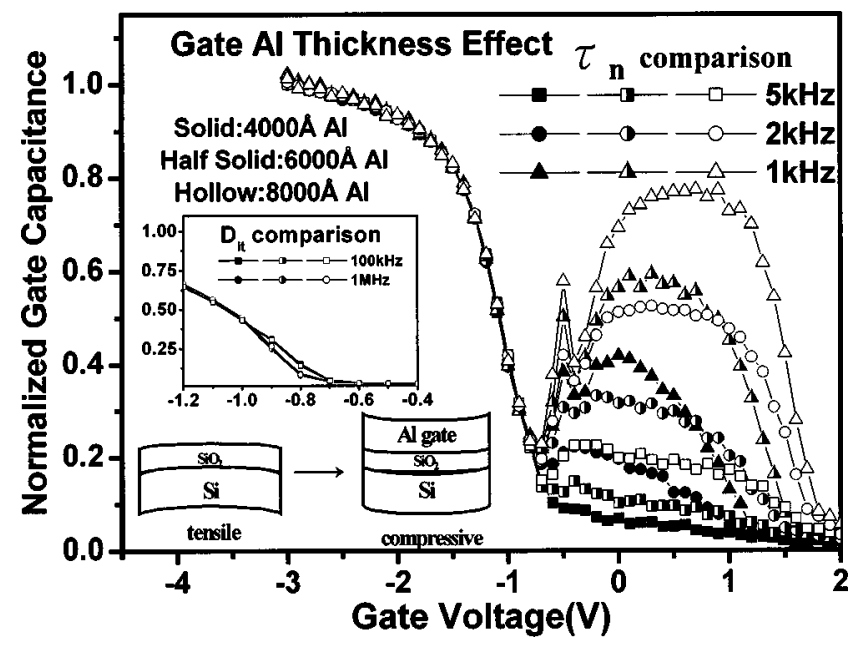

FIG. 5. The normalized $C-V$ curves for samples with different gate $\mathrm{Al}$ thicknesses measured under illumination at various frequencies, using for $\tau_{n}$ comparison. The inset shows dark $C-V$ curves in depletion region measured at two different frequencies, using for $D_{\text {it }}$ comparison. The extra schematic diagram shows the stress effect of gate $\mathrm{Al}$ deposition on an MOS structure. which in turn leads to a higher $J_{\mathrm{dg}}$ and thus $J_{\text {sub }}$, as observed in Fig. 4. The trap-related effects influence the $J_{\text {sub }}$ behavior of devices for different gate Al thickness. On the other hand, the band gap-related effect may be also responsible for the increase in $J_{\text {sub }}$. For a certain oxide thickness, the Si wafer is generally under tensile stress. After the gate $\mathrm{Al}$ deposition, if the Al film is sufficiently thick, the chip curvature changes from convex to concave, ${ }^{9}$ as shown by the schematic diagram in Fig. 5, such that the silicon wafer is now under compressive stress. A thicker gate $\mathrm{Al}$ applies a larger compressive stress to $\mathrm{Si}$, decreasing $\mathrm{Si}$ band gap, thereby increasing the $n_{i}$ and $J_{\text {sub }}$ of the devices.

In conclusion, $J_{\text {sub }}$ of an MOS structure changes in three ways with the thickness of each component. (1) $J_{\text {sub }}$ increases with the oxide thickness, the mechanism for which is suggested to be dominated by the trap-related effect. (2) $J_{\text {sub }}$ of a device on a thick wafer is more sensitive to a change in the oxide thickness than that of a device on a thin wafer. The mechanism is suggested to be dominated by the band gaprelated effect. (3) $J_{\text {sub }}$ increases with the gate Al thickness. Both the trap-related and band gap-related effects are responsible for this observation. The substrate injection current is very sensitive to the thickness of oxide, $\mathrm{Si}$ wafer, and gate $\mathrm{Al}$, and is useful for examining the effects of stress in $p$-type MOS structures.

The authors would like to thank the National Science Council of the Republic of China for financially supporting this research under Contract No. NSC 91-2215-E-002-023.

${ }^{1}$ S. M. Hu, J. Appl. Phys. 70, R53 (1991)

${ }^{2}$ D. Chin, S. Y. Ho, S. M. Hu, R. W. Dutton, and J. L. Moll, Tech. Dig. - Int. Electron Devices Meet. 1982, 228 (1982).

${ }^{3}$ J. Y. Yen and J. G. Hwu, Appl. Phys. Lett. 76, 1834 (2000).

${ }^{4}$ B. S. H. Royce, IEEE Trans. Compon., Hybrids, Manuf. Technol. 11, 454 (1988)

${ }^{5}$ H. Lee, Y. Huh, J.-S. Goo, S.-D. Lee, D. Yang, and W. Kim, Tech. Dig. Int. Electron Devices Meet. 1995, 653 (1995).

${ }^{6}$ C. H. Bjorman, J. T. Fitch, and G. Lucovsky, Appl. Phys. Lett. 56, 1983 (1990).

${ }^{7}$ A. Hamada, T. Fursawa, N. Saito, and E. Takeda, IEEE Trans. Electron Devices 38, 895 (1991).

${ }^{8}$ H. Miura, S. Ikeda, and Suzuki, Tech. Dig. - Int. Electron Devices Meet. 1996, 743 (1996).

${ }^{9}$ V. Zekeriya and T. P. Ma, J. Appl. Phys. 56, 1017 (1984).

${ }^{10}$ T. B. Hook and T. P. Ma, Appl. Phys. Lett. 48, 1208 (1986).

${ }^{11}$ C. W. Liu, M. H. Lee, C. F. Lin, I. C. Lin, W. T. Liu, and H. H. Lin, Tech. Dig. - Int. Electron Devices Meet. 1999, 749 (1999).

${ }^{12}$ N. Yang, W. K. Henson, and J. J. Wortman, IEEE Trans. Electron Devices 47, 1636 (2000).

${ }^{13}$ S. M. Sze, Physics of Semiconductor Devices (Wiley, New York, 1981).

${ }^{14}$ M. Y. Doghish and F. D. Ho, IEEE Trans. Electron Devices 39, 2771 (1992).

${ }^{15}$ C. C. Hong and J. G. Hwu, Appl. Phys. Lett. 79, 3797 (2001).

${ }^{16}$ C. H. Lin, B. C. Hsu, M. H. Lee, and C. W. Liu, IEEE Trans. Electron Devices 48, 2152 (2001).

${ }^{17}$ J. Bardeen and W. Shockley, Phys. Rev. 80, 72 (1950).

${ }^{18}$ J. L. Su, C. C. Hong, and J. G. Hwu, J. Appl. Phys. 91, 5423 (2002).

${ }^{19}$ K. J. Yang and C. Hu, IEEE Trans. Electron Devices 46, 1500 (1999).

${ }^{20}$ E. H. Nicollian and J. R. Brews, MOS Physics and Technology (Wiley, New York, 1982).

${ }^{21}$ G. Charitat and A. Martinez, J. Appl. Phys. 55, 909 (1984). 
Applied Physics Letters is copyrighted by the American Institute of Physics (AIP). Redistribution of journal material is subject to the AIP online journal license and/or AIP copyright. For more information, see http:/ojps.aip.org/aplo/aplcr.jsp

Copyright of Applied Physics Letters is the property of American Institute of Physics and its content may not be copied or emailed to multiple sites or posted to a listserv without the copyright holder's express written permission. However, users may print, download, or email articles for individual use. 